\title{
Co-rotating equal strength vortex pair merging in channel flow with pressure gradient environment
}

\author{
Yuan $\mathrm{Yi}^{1, \text { a }}$, Peiqing Liu ${ }^{1, \mathrm{~b}}$ and Tianxiang $\mathrm{Hu}^{1, \mathrm{c}}$ \\ ${ }^{1}$ Beihang University, Beijing, China \\ aby1205188@buaa.edu.cn, blpq@buaa.edu.cn, ctianxiang.hu@buaa.edu.cn
}

\begin{abstract}
Keywords: vortex merger, channel flow, pressure gradient
Abstract. Experiments were conducted in order to understand the merging process of co-rotating equal strength vortex pair in channel flow with different pressure gradient environment. Cross-flow velocity measurements were performed at a series of downstream locations under zero, favorable and adverse pressure gradient conditions. The main finding is that the pressure gradient has a vastly effect on the merging process. As the merging environment changed from favorable pressure gradient (FPG) to adverse pressure gradient (APG), the increasing of the vortex spacing was observed in the first diffusive stage, which lead to a delayed convective stage. However, at the convective stage where the spacing of vortex pair center decreased, the decreasing process gradually speed up from FPG to APG accompanied with a faster vortices transportation and diffusion process so the merging process is accordingly accelerated. On the other hand, the vortex orientation difference was not as conspicuous as the vortex center separation distance.
\end{abstract}

\section{Introduction}

The channel flow has been a long-studied research problem with significant engineering applications and channel flow control with passive methods has been studied excessively in both experimental and numerical approaches in the last several decades[1][2]. Streamwise vortices generated by longitudinal vortex generators(LVGs) such as adding fins or baffles are receiving more and more attention both in laminar and turbulent flow regimes for their strong perturbation in the boundary layer and long persistence over streamwise distances [3].

Tiggelbeck [4] experimentally studied the heat transfer enhancement and drag with double rows of delta winglets. The result showed that the critical angle of attack to create longitudinal vortices behind the second row was smaller than that behind the first one, and heat transfer coefficient and drag were increased by $80 \%$ and $160 \%$. Apart from channel flow control, there are more LVGs applications in fluid machinery and engineering like wind turbine. Suarez[5] simulated the passive control using streamwise vortices and Sullivan[6] tested the wind turbine with LVGs arry, both their results proved performance improvement.

For different types of vortex generator could be mounted in various position, the co-rotating vortex pair system emerges from the interactions between the complicated streamwise vortex system consisted of both individual and between LVGs. The equal-strength co-rotating vortex merging process has been divided into three stages by Meunier [7], while Cerretelli [8] added the fourth stages which eventually describe the process as first diffusive stage, convective stage, second diffusive stage and merged diffusive stage. In the first diffusive stage, the co-rotating vortex pair undergoes diffusive growth, with the vortices rotating around each other due to mutual induction and the vortex separation remains constant. When the vortex grows large enough, the antisymmetric vorticity induced by another vortex gradually diffuses into the outer region leading to the onset of the convective merger, causing the two vortices to move rapidly towards each other. Hopfinger [9] and Meunier [7] studied the critical core size above which merging takes place and found the critical ratio of radius and separation for merging was around 0.3. Crow [10] and Leweke [11] observed the long-wavelength and shorter-wave elliptical instabilities in instabilities study of vortex merging.

It is well known that as the flow performance improves by the streamwise vortex system, the corresponding pressure drop also becomes tremendous, which means the co-rotating vortex merging in a channel flow with streamwise pressure variation [2][5]. As a result, although vortex merging has 
been studied in many aspects, the present study mainly focus on the merging mechanism or partial flow field transform, which means the merging process proceeded in relatively simple conditions. This work intended to study the process under the influence of adverse pressure gradient so a foundation would be laid for further study for vortex merging in more complex environment.

\section{Experimental system and techniques}

Experiments were conducted in the low Reynolds straight-flow free surface water tunnel at BeiHang University with the test section size of $400 \mathrm{~mm}(\mathrm{~W}) \times 600 \mathrm{~mm}(\mathrm{H}) \times 4500 \mathrm{~mm}(\mathrm{~L})$. In this experiment, the test section is constituted with three parts as it was showed in Fig. ${ }^{\circ}$. The first part is a $400 \mathrm{~mm}$ long contraction section to lift the incoming flow; followed with a $600 \mathrm{~mm}$ long straight section where the vortex pair was generated; the last part is a $2200 \mathrm{~mm}$ long plate with adjustable divergence angle. The divergence angle could be set as $2 \mathrm{deg}$ upward, $0 \mathrm{deg}$ or $3 \mathrm{deg}$ downward so the favorable, zero or adverse pressure gradient condition was generated correspondingly. For simplified description, favorable, zero and adverse pressure condition was shorted as FPG, ZPG and APG.The coordinate system is as follows: the $\mathrm{X}$-axis is in the downstream direction; the Z-axis is vertical along with the depth direction, while the Y-axis is perpendicular to the flow direction and horizontal; original point was the center of the final plane of the straight section.

The co-rotating vortex pair was generated by two horizontally placed, submerged rectangular wings with opposite angle of attack. The airfoil section is NACA0012 with $0.1 \mathrm{~m}$ chord length and $0.15 \mathrm{~m}$ span and rounded at wing-tip edges. For the convenience of description, the vortex moved downward from the beginning was named vortex1 while the other was named vortex 2 .

Quantitative measurements of velocity fields were obtained using a 2-D Digital Particle Image Velocimetry(DPIV) system produced by Dantec, which produces up to $200 \mathrm{~mJ}$ pulses from a pair of mini Nd:YAG laser heads at a rate of 1pairs/second for 300 image for one test plane. Images were taken with $2048 * 2048$ pixel digital CCD camera. Adaptive-Correlation was performed on 300 image pairs with a $32 * 32$ pixel interrogation window. This produced a vector spatial resolution at the measurement stations $\mathrm{x} / \mathrm{c}=0 \sim 20$ for with distance interval of 0.5 . Uncertainty in the velocity data was estimated to be $2 \%$.

A co-rotating vortex pair is characterized by the following parameters as showed diagrammatically in Fig2.The major parameters like a, D, or $\theta$ mentioned above had a highly dependency on the specific definition of the vortex core center position. Since the instantaneous vortices distribution in co-rotating vortex merging is quite irregular, the vortex center coordinate is defined by Eq.(1) and (2). Considering the accuracy and consistency of this method, Q value (see Eq.(3)) is introduced so that $\mathrm{A}$ (the integration area) could be defined by the region where the $\mathrm{Q}$ value is more than $5 \%$ of the maximum. The averaged center position is the final vortex center coordinate.

$$
\begin{aligned}
& Y_{s}=\frac{\int x \omega d A}{\Gamma} \\
& Z_{s}=\frac{\int z \omega d A}{\Gamma} \\
& Q=\frac{d v}{d y} \frac{d w}{d z}-\frac{d v}{d z} \frac{d w}{d y}
\end{aligned}
$$

To minimize the interaction between the vortex generators as well as ensure the merging process was observed in the test section, all the parameters were carefully selected. In this experiment, at the first measured profile, which is the end plane of the straight section, the effected velocity $\left(\mathrm{U}_{\mathrm{EFF}}\right)$ was $0.107 \mathrm{~m} / \mathrm{s}, \Gamma$ was $0.00191 \mathrm{~m} 2 / \mathrm{s}, \theta$ was $0 \mathrm{deg}, \mathrm{b}$ was $0.0456 \mathrm{~m}$. The water temperature was $16{ }^{\circ} \mathrm{C}$, correspondingly $\operatorname{Re}_{\Gamma}$ was 1717, Reynolds number based on chord was 9620. 


\section{Results and discussion}

To describe the pressure gradient difference of the three testing environment, the cross plane center velocity is measured before the vortex generators were mounted. The velocity magnitude distribution along with the $\mathrm{X}$ direction is diagramed in Fig. ${ }^{\circ}$.In the test section, the flow speed maintains constant under ZPG condition. Since the adjustable plate is placed with a certain angle, the flow either speed up or speed down so that GPG condition or ZPG condition could be created. In the end of the test section, although the divergence is slightly changed, the flow rate difference can reach nearly as much as $30 \%$.

The cross-flow PIV measurements of co-rotating vortex pair merging process are shown in Fig. ${ }^{\circ} 4$ with normalized time-averaged vortices distribution. The vortices contour is continuously translated from 0 to 5, where the positive vortices rotate counter-clockwise. Since the pressure gradient environment is induced by the adjustable plate in test section, the vortex pair center correspondingly moves upward or downward in FPG condition or APG condition. The ZPG merging process provide a baseline for FPG and APG. During the early stage of the merging process when $\mathrm{x} / \mathrm{c}<5$, the existence and decay of the vortex generator wake can be clearly noticed while neither the vortices strength nor orientation angle has much difference on the whole. Fig. ${ }^{\circ} 4$ shows that although the orientation of FPG and APG reach an approximately value lower than ZPG condition at $\mathrm{x} / \mathrm{c}=17$, the FPG maintains well with ZPG at $\mathrm{x} / \mathrm{c}=12$, which means the delaying of orientation in FPG appears at a later stage of the merging process. As for vortices distribution, all three merging process started with two separate concentrated vortexes, developed with vortices diffusion, decay and transformation, ended with one scattered vortex. Due to the effect of pressure gradient, the extreme vortices value of APG is lower than the other two at $\mathrm{x} / \mathrm{c}=17$. It can be seen that the vortex distribution is successively concentrated from FPG to APG at $\mathrm{x} / \mathrm{c}=19$ and $\mathrm{x} / \mathrm{c}=20$. For $\mathrm{APG}$ condition, the rounded process has almost completed in the merged diffusive stage.

The distance between vortex cores reveals the quantitative merging process of vortex pair approaching each other. For typical free equal strength co-rotating vortex pair(like ZPG condition in this experiment), although it is believed the vortex separate distance should be appeared as a steady first diffusive stage where the vortex pair maintains at a certain distance, previous experiments has observed the fact that the vortex pair would departure $10 \%$ to $20 \%$ of its initial separation for a certain distance in this stage and attributed this phenomenon to the disturbance induced by the wake of vortex generators[7,8]. Fig. ${ }^{05}$ shows the evolution of the vortex separation in all three pressure gradient environment. There is not much difference for all the separation continues growing up until $\mathrm{x} / \mathrm{c}=3$ where FPG condition steps into the convective stage. The start point of convective stage for ZPG condition is $\mathrm{x} / \mathrm{c}=4$ while the APG condition is delayed to $\mathrm{x} / \mathrm{c}=5$. When it comes to the convective stage, the vortex separation continuously decrease approximately linearly under ZPG condition. Different from FPG case which seems also linearly decreases with a lower rate than ZPG in the whole convective stage, the decreasing speed in APG case is obviously accelerated during the second half of the convective stage. As a result, although the start point of the convective stage is gradually postponed from FPG to APG condition, it seems all the three ended at the same position around $\mathrm{x} / \mathrm{c}=17$. When it comes to the second diffusive stage, compared with ZPG case, the merging process is shorted in APG condition while the separated vortex can still be recognized at a lateral streamwise position. In general, the start position of the convective stage is correspondingly delayed from FPG to APG and comes to the same position in the end, but on the contrary to the final position of the whole merging process.

Compared with the separation distance, there are less noteworthy features in vortex pair orientation (Fig. ${ }^{\circ}$ ). Under the ZPG condition, the orientation angle increases linearly in the first diffusive stage and increasing speeds up afterwards. On the whole, both the APG and FPG slowed down the increase of pair orientation. The difference was unrecognizable before $\mathrm{x} / \mathrm{c}=6.5$ where the orientation was around 90 degree, after which the APG orientation was gradually falling behind; the FPF orientation maintained well with ZPG and did not come close to APG until $\mathrm{x} / \mathrm{c}=13$.

Due to the orbital revolution of the vortex pair, both vortex moved clockwise. Vortex center track is diagramed in two different ways so that the vortex movement can be properly featured. In Fig. ${ }^{\circ}$, (a) 
and (b) individually demonstrate the vortex movement with projected coordinate on crossflow plan, (c) and (d) demonstrate the horizontal and vertical coordinate along with the flow direction. From Fig. ${ }^{\circ}$ (a) and (b), it can be seen that the two vortex rotated with each other and horizontally moves into a closer position to the crossflow centerline compared with initial position. Unlike the centrosymmetric vortex track under ZPG condition, there is significant difference in vertical movement under both the FPG and APG environment. It is noteworthy that both vortex 1 and vortex 2 track in APG condition maintains with ZPG in a short distance while the FPG deviated from the begging with a smaller deflection angle. The horizontal movement in Fig. ${ }^{\circ} 7$ (c) shows that the vortex pair center remains in the middle plane of the test section all a long which means none of the merging process was affect by the ground effect. Fig. ${ }^{\circ} 7$ (c) also reveals the fact that the horizontal difference is not notable until the vortex moved across the middle plane. As it is mentioned in Fig. ${ }^{\circ}$, due to the adjustable plate, the vortex pair center seems linearly moved upward or downward vertically shown in Fig. ${ }^{\circ} 7$ (d).

Turbulence plots showing the development of the equal strength vortex pair along with the flow direction can be found in Fig. ${ }^{\circ} 8$. Under all three conditions, there is a common developing phenomenon that from $\mathrm{x} / \mathrm{c}=9$ to $\mathrm{x} / \mathrm{c}=20$ the vortex center turbulence decays all the way downstream while the background turbulence has a rising and decreasing process. For the concentrated vortex in convective stage, the evident turbulence area surrounded with the vortex center indicate the active wondering of each vortex. The ZPG condition in Fig8 clearly demonstrate that the two arm-like high turbulence area stretch from the endpoint of the vortex pair long axis and spread throughout the whole area form $\mathrm{x} / \mathrm{c}=9$ to $\mathrm{x} / \mathrm{c}=13$, as the convective stage comes to the end around $\mathrm{x} / \mathrm{c}=17$, the arm-like area shrinks and the back ground turbulence decays since to the end.

At the same $\mathrm{x} / \mathrm{c}$ position in Fig. ${ }^{\circ} 8$, the background turbulence successively increase from APG to FPG, which is conductive to the merger. As for the high turbulence area near vortex center, since the pressure gradient has a remarkable influence on vortex behavior, the concentration of the high turbulence area around vortex center is ascending form FPG to APG. Although the concentration is ascending for FPG to APG, the overall strength does not has the same tendency. From $\mathrm{x} / \mathrm{c}=9$ to $\mathrm{x} / \mathrm{c}=13$, the APG condition has the highest value of the turbulence strength near vortex center, correspondingly the center distance decreasing speed ascendingly ranked from FPG to APG from with significant difference at the same interval in Fig5, which means the obvious vortex wonder with efficient transport helps with the merger in convective stage. On the other hand, at $\mathrm{x} / \mathrm{c}=17$, the FPG condition has relatively concentrated high turbulence vortex center areas lager than the other two and maintains the phenomenon to the end at $\mathrm{x} / \mathrm{c}=20$. On the whole, Fig8 reveled the fact that with active vortex wonder and efficient energy transport the merger can be greatly promoted.

The above content mainly compared the merging process at the same downstream position where the center distance and orientation angle are different under each testing condition and come to the conclusion that the merging process changed dramatically in vortex center separation decreasing speed at the middle of the convective stage from $\mathrm{x} / \mathrm{c}=8$ to $\mathrm{x} / \mathrm{c}=14$.

The physical mechanism of equal strength co-rotating vortex pair merging has been extensively studied for many years. One of the mainstream theories presented by C.Cerretelli and C.H.K. [8] Williamson explained the merging mechanism with antisymmetric vortices and symmetric vortices, and it was believed the form of the antisymmetric vortices induced a velocity field pushes the two vortex together. To physically understand the pressure gradient effect on vortex merging, the origin of coordinates is repositioned to the vortex pair center so that the vortices field can be resolved to symmetric vortices field and antisymmetric vortices field.

Fig. ${ }^{\circ} 9$ and Fig. ${ }^{\circ} 10$ show two sets of normalized antisymmetric vortices $\left(\omega_{\mathrm{A}}\right)$ distribution selected with the same D/D0. For the convenience of comparison, the coordinate system is rotated so the three vortex center pair appear as one. Unlike the antisymmetric vortices filed showed in C.Cerretelli and C.H.K. Williamson's work which has four relatively concentrated vortex with antisymmetric distribution, there seems to be 8 concentrated vortex and 4 looming vortex sheet as demonstrated.

Fig. ${ }^{\circ} 9$ compares the pressure gradient effect on vortex merger when it reaches the same vortex separation of $\mathrm{D} / \mathrm{D}_{0}=0.04923$ at $\mathrm{x} / \mathrm{c}=8.5,10$ and 11.5 correspondingly under $\mathrm{FPG}, \mathrm{ZPG}$ and $\mathrm{APG}$ 
condition. Skewsymmetric vortices contour distribute from inside to outside represent FPG to APG condition in Fig. ${ }^{\circ} 9$ (a), which means the APG condition has the strongest diffusion of the three. The contour distribution in Fig. ${ }^{\circ} 9$ (a) also show the difference between FPG and ZPG mainly come from the second and fourth quadrant, while the difference between APG and ZPG mainly come from the first and third quadrant. Normalized antisymmetric vortices distribution can be found in Fig. ${ }^{\circ} 9$ (b), (c) and (d) with velocity back calculated which indicate the existence of two obvious counter-rotating vortex pairs outside the vortex center region. As described earlier, there are two pairs of concentrated counter-rotating vortex pairs around each vortex center, half surrounded by two looming counter-rotating vortex sheet which has the same direction with concentrated vortex nearby. It is clear that the APG has the strongest and largest area of concentrated vortex at correspondingly position which means the vortex has the highest transformation level and the highest induced velocity field to push the vortex centers together.

A lateral state in Fig10 was performed in exactly the same manner as Fig. ${ }^{\circ} 9$ so the evolution of $\omega_{\mathrm{A}}$ could be illustrated. The plots in Fig. ${ }^{\circ} 9$ are all near to the end position of the convective stage where the vortex center separation changing tendencies join together as scattered in Fig5. Despite the inconspicuous distinction in contour line in Fig. ${ }^{\circ} 10$ (a) and looming vortex sheet in Fig10 (b), (c) and (d), Fig. ${ }^{\circ} 10$ explains the same pressure gradient effort on the vortex merger.

The evolution of ${ }^{\omega_{A}}$ can be explained by comparing Fig. ${ }^{\circ}$ and Fig. ${ }^{\circ} 10$ accordingly. As the vortex pair centrosymmetric transformation develops with the individual vortex resembling an ellipse in shape, the concreted counter-rotated vortex pairs beside the vortex centers enlarged in area while the two between the centers shrink with a lower value, also the looming vortex sheet become less recognizable. In conclusion, during the convective stage, the adverse pressure gradient makes a contribution in diffusion and transformation for both the vortex pair and individual vortex while the favorable pressure gradient works in an opposite way.

\section{Conclusions}

The dynamic interactions of co-rotating equal strength vortex pair merger was explored under three different pressure gradient environment. FPG (Favorable Pressure Gradient), ZPG (Zero Pressure Gradient) and APG (Adverse Pressure Gradient) environment were produced to replicate the complex merging process in practical application flows.

It turned out that during the four stages of the merger, compared with ZPG condition, although the first diffusive stage is extended in APG condition and shorted in FPG condition, the second diffusive stage ended around the same position, which means an accelerated or decelerated merging process correspondingly. This tendency continues to the second diffusive stage where the two vortex merged as one and is expected to be sustained to the end. The pressure gradient environment has less impact on orientation, for the orientation angle under both APG and FPG falling behind from different position by less than 90 degrees.

The experimental result proved that the pressure gradient mainly affected merger by changing the strength of vortices transportation and diffusion, the combined effect can greatly affect the convective stage. When it comes to the second diffusive stage where the viscous diffusion plays a major role, only the diffusion effect can be observed. On the whole, the merging process could be accelerated or decelerated under APG or FPG condition, and it can be predicted that the merger difference would be enlarged if the wake effect could be eliminated in the first diffusive stage.

\section{References}

[1] Reddy S C, Dan S H. Energy growth in viscous channel flows[J]. Journal of Fluid Mechanics, 1993, 252(252):209-238.

[2] Piomelli U, Liu J. Large - eddy simulation of rotating channel flows using a localized dynamic model[J]. Physics of Fluids, 1995, 7(4):839-848. 
[3] Jacobi A M, Shah R K. Heat transfer surface enhancement through the use of longitudinal vortices: A review of recent progress[J]. Experimental Thermal \& Fluid Science, 1995, 11(3):295-309.

[4] Tiggelbeck S, Mitra N K, Fiebig M. Experimental investigations of heat transfer enhancement and flow losses in a channel with double rows of longitudinal vortex generators[J]. International Journal of Heat \& Mass Transfer, 1993, 36(9):2327-2337.

[5] Suarez J M, Flaszyński P, Doerffer P. Streamwise vortex generator for separation reduction on wind turbine profile[J]. 2016, 760(1):012018.

[6] Sullivan T L. Effect of vortex generators on the power conversion performance and structural dynamic loads of the Mod-2 wind turbine[J]. National Aeronautics \& Space Administration Report, 1984.

[7] Meunier P, Leweke T. Three-dimensional instability during vortex merging[J]. Physics of Fluids, 2001, 13(10):2747-2750.

[8] Cerretelli C, Williamson C H K. The physical mechanism for vortex merging[J]. Journal of Fluid Mechanics, 2003, 475(475):41-77.

[9] Hopfinger E J, Heijst G J F V. Vortices in Rotating Fluids[J]. Annual Review of Fluid Mechanics, 1993, 25(25):241-289.

[10] Crow S C. Stability theory for a pair of trailing vortices[J]. Aiaa Journal, 1970, 8(12):2172-2179.

[11]Leweke T, Williamson C H K. Cooperative elliptic instability of a vortex pair[J]. Journal of Fluid Mechanics, 1998, 360(360):85-119.

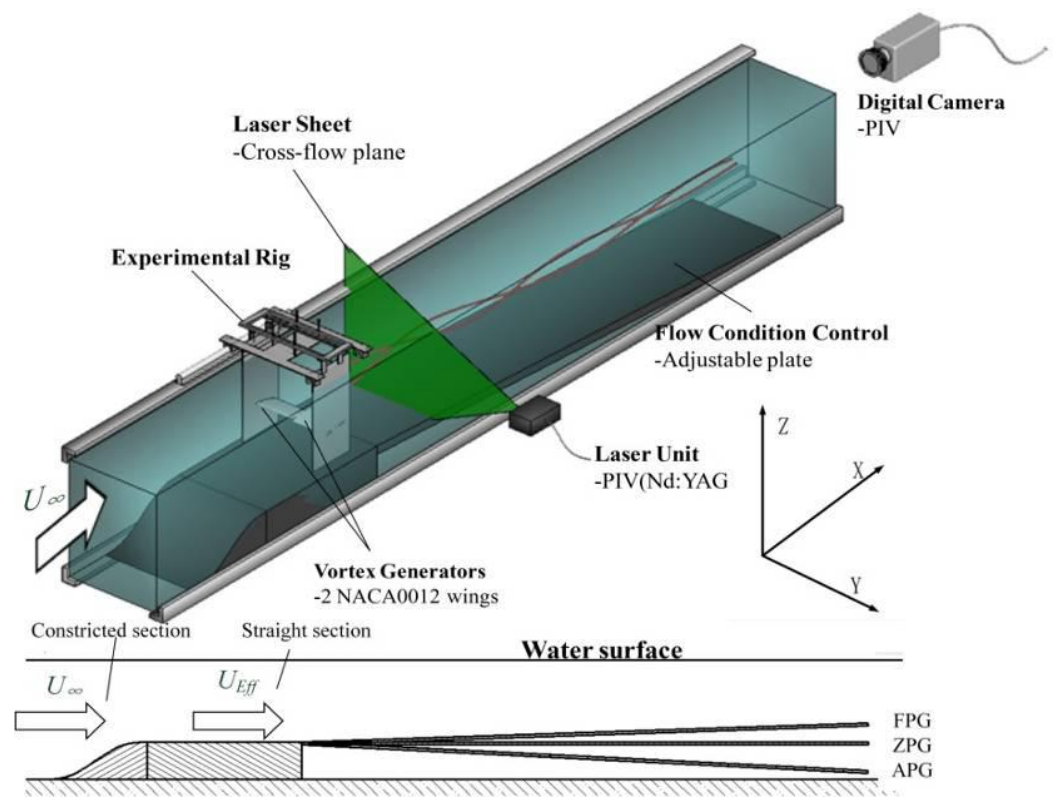

Fig. ${ }^{\circ} 1$ experiment setup 


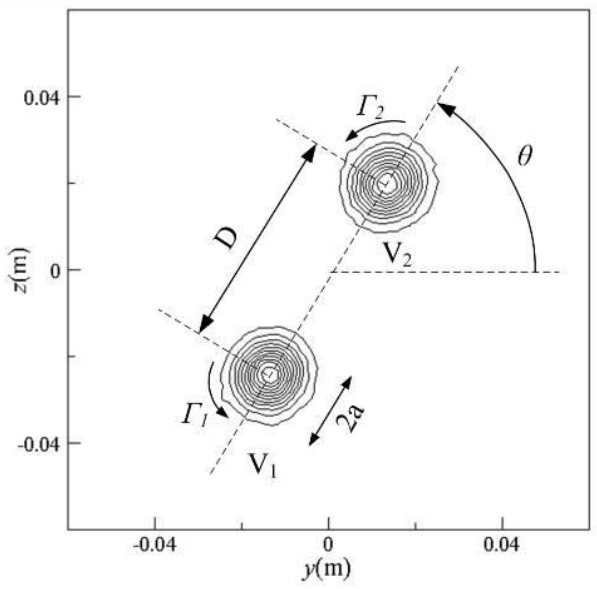

Fig. ${ }^{2}$ Definition of the parameters characterizing the vortex pair

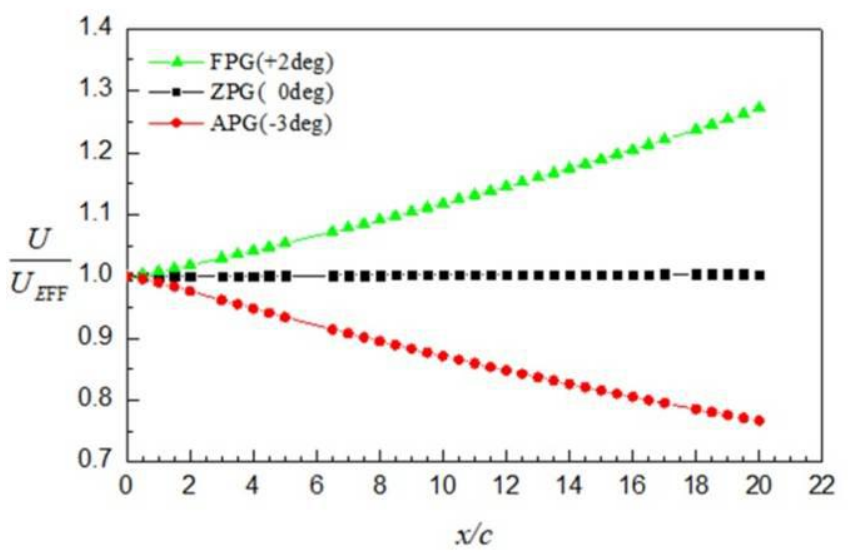

Fig. ${ }^{\circ} 3$ Cross-plan center velocity distribution at different streamwise position

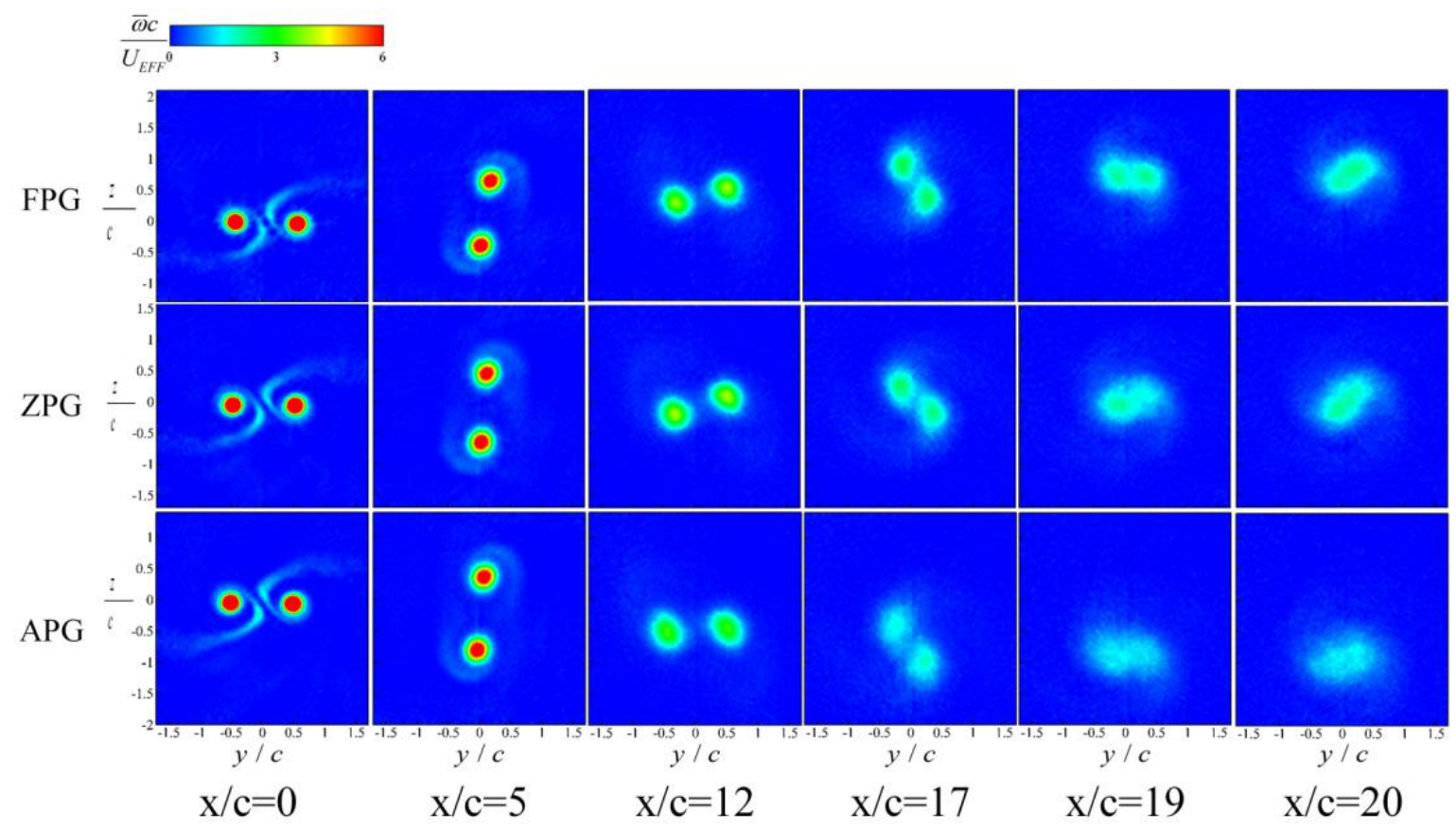

Fig. ${ }^{4}$ Normalized time-averaged vorticity distribution at different streamwise position.

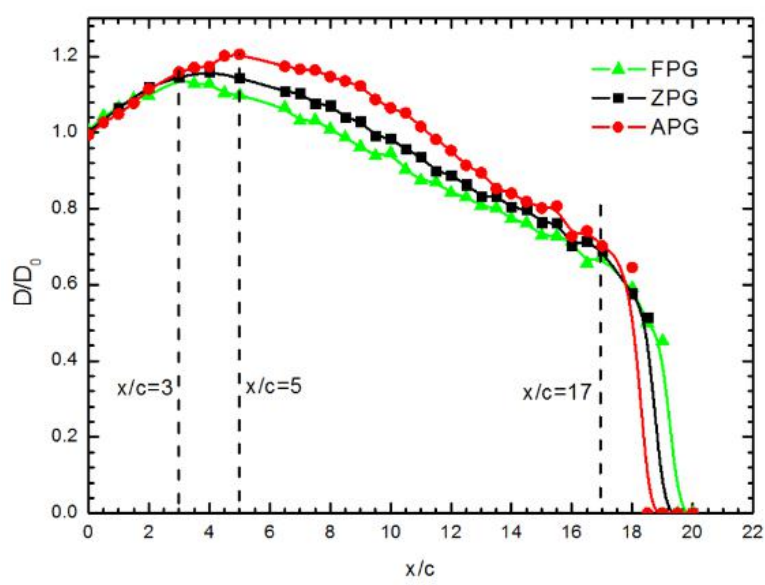

Fig. ${ }^{\circ} 5$ Evolution of the vortex separation

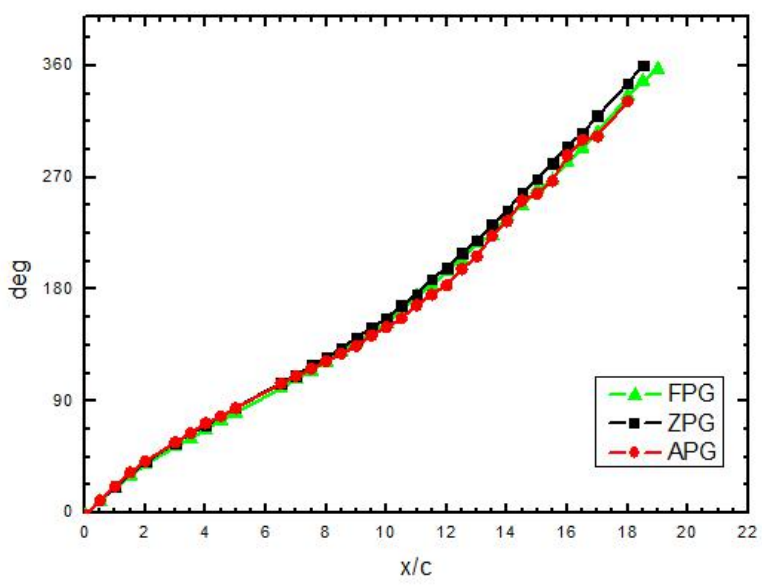

Fig. ${ }^{\circ} 6$ Evolution of the vortex pair orientation 
(a)

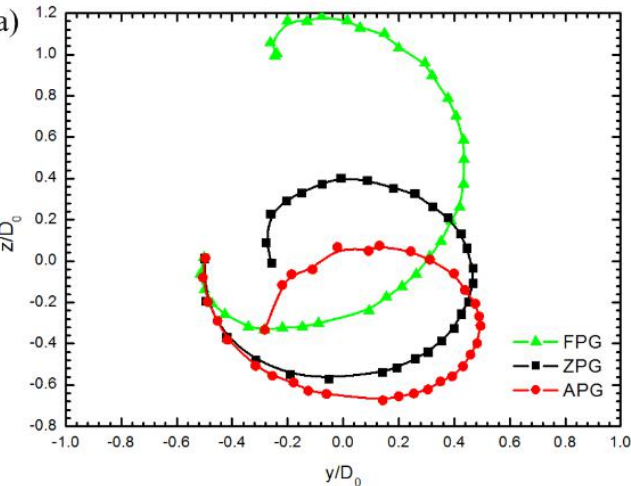

(c)

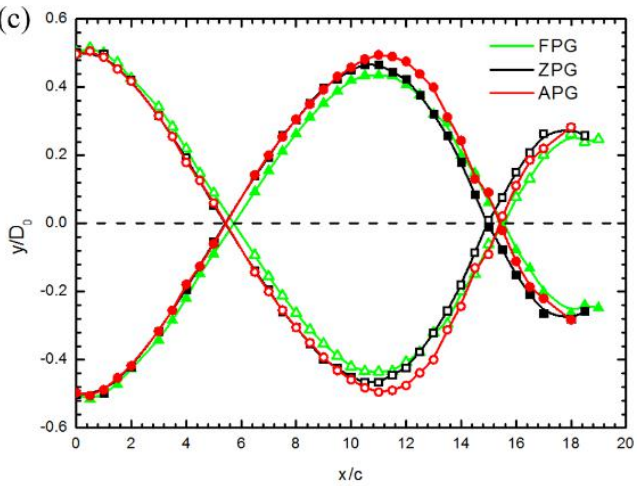

(b)

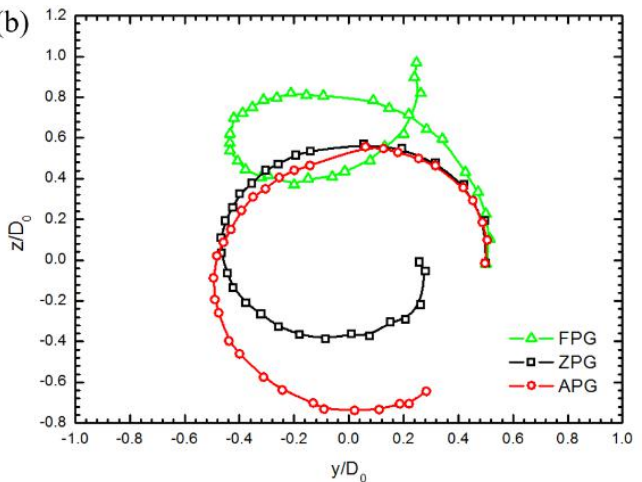

(d)

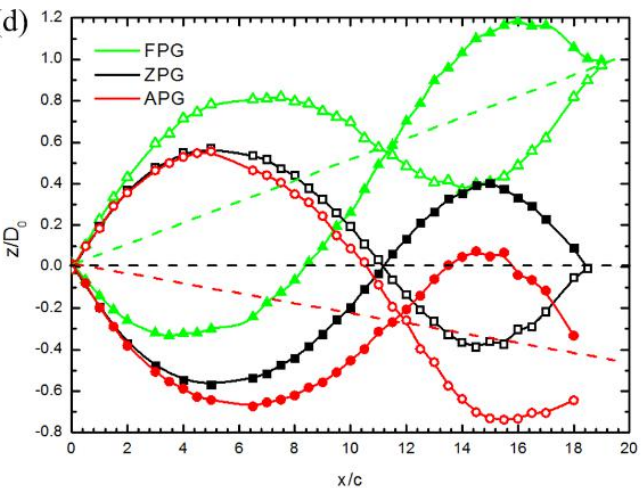

Fig. ${ }^{\circ} 7$ Evolution of the normalized vortex center coordinate.

(a) (b)Crossflow plan projected coordinate of vortex 1 and vortex 2 .

(c) Horizontal coordinate. (d) Vertical coordinate.

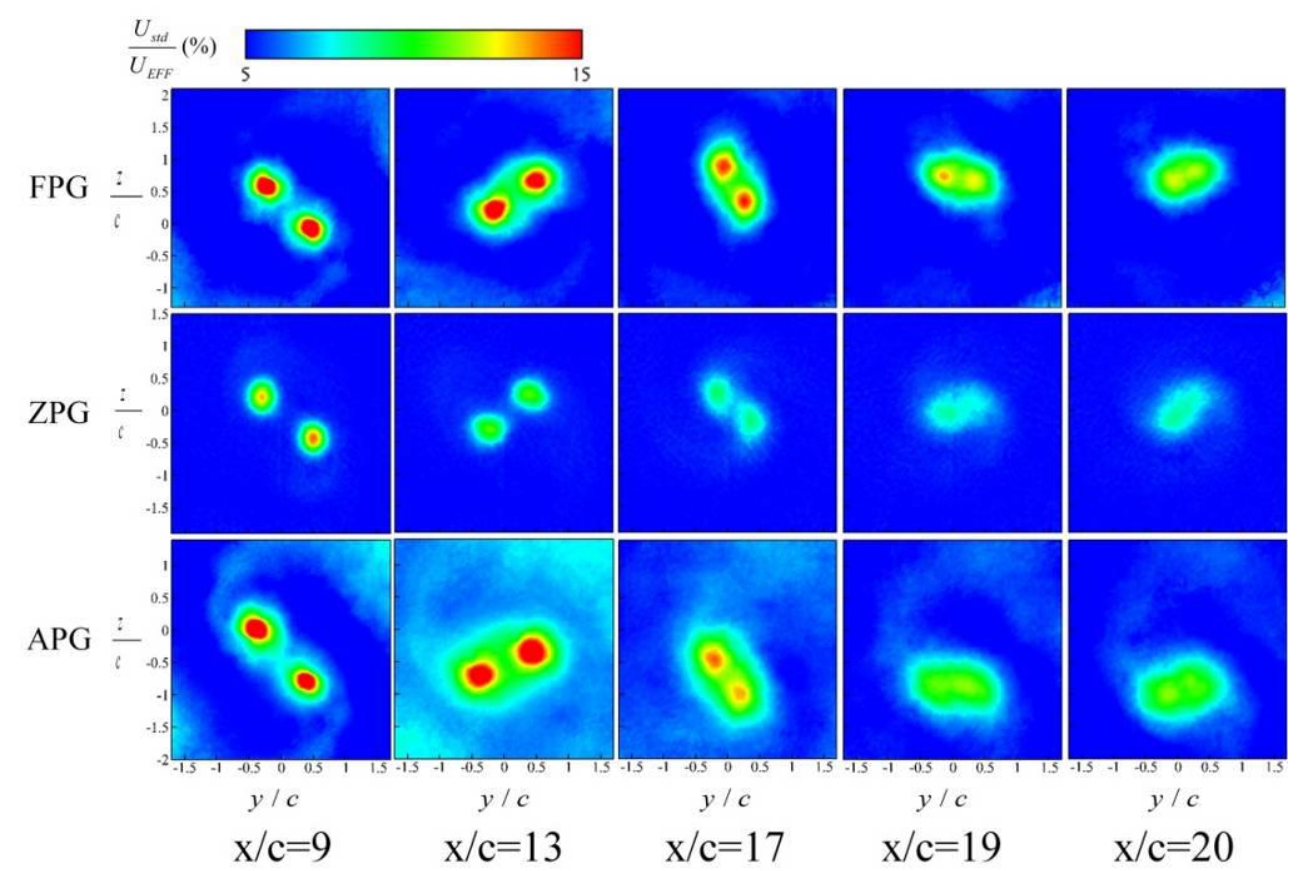

Fig. ${ }^{\circ} 8$ Turbulence intensity distribution at different streamwise position. 

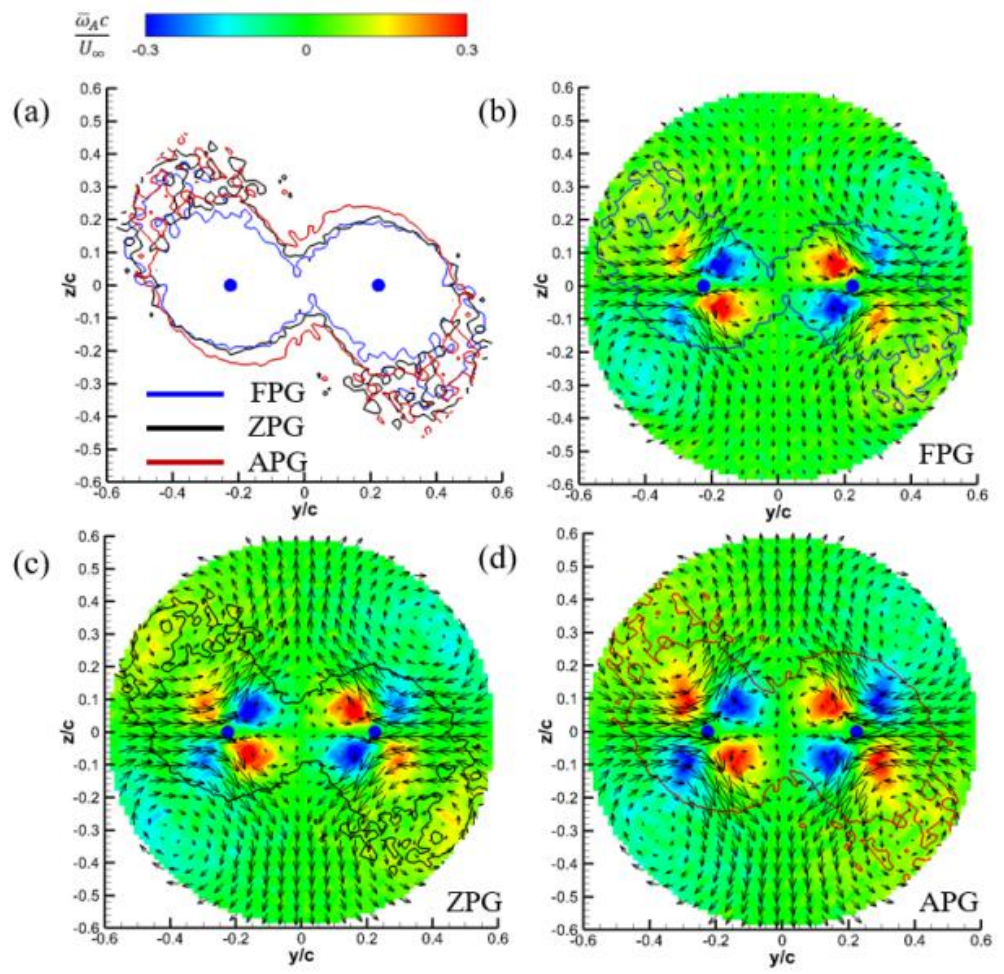

Fig. ${ }^{\circ} 9 \mathrm{D} / \mathrm{D}_{0}=0.04923$; (a) Skew symmetric vortices contour $\omega_{\mathrm{S}} \mathrm{C} / \mathrm{U}_{\mathrm{EFF}}=0.17$, (b)FPG x/c=8.5, (c)ZPG x/c=10, (d)APG x/c=11.5

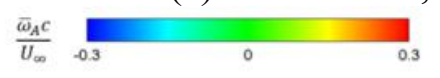

(a)

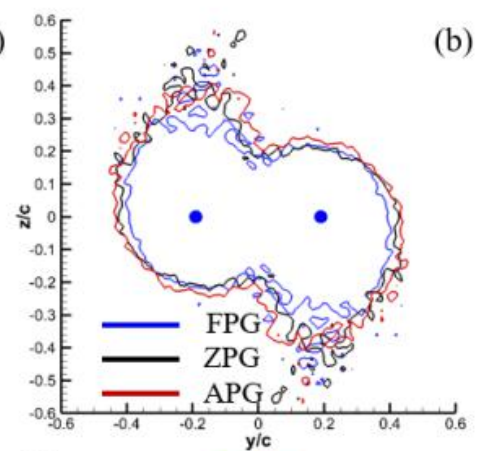

(c)

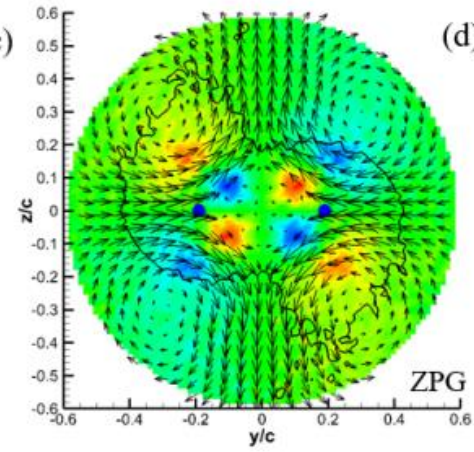

(b)

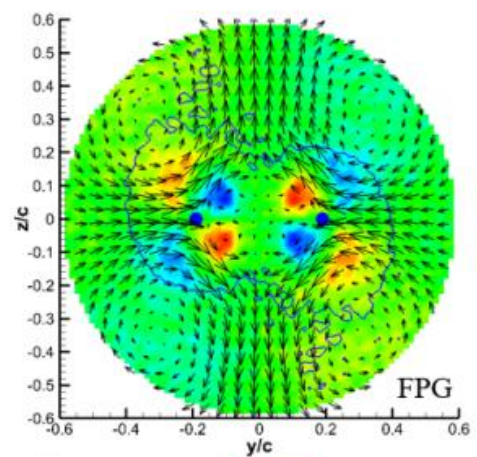

(d)

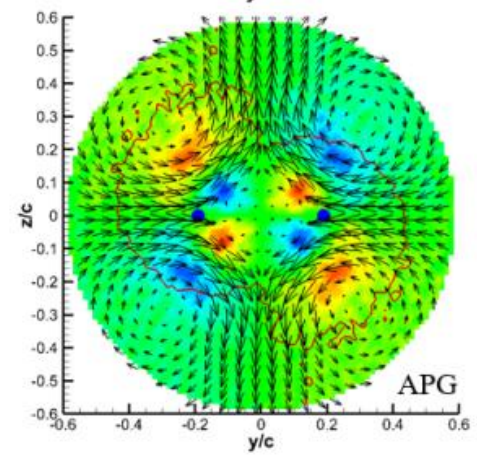

Fig. ${ }^{\circ} 10 \mathrm{D} / \mathrm{D}_{0}=0.04171$; (a) Skew symmetric vortices contour $\omega_{\mathrm{S}} \mathrm{C} / \mathrm{U}_{\mathrm{EFF}}=0.17$, (b)FPG x/c=12.5, (c)ZPG x/c=13.5, (d)APG x/c=14 